Schweiz. Gastroenterol. $2021 \cdot 2: 56-66$ https://doi.org/10.1007/s43472-021-00040-4 Accettato: 26.42021

Pubblicato online: 1.72021

○ Der/die Autor(en) 2021

\author{
Benedetta Terziroli Beretta-Piccoli ${ }^{1,2,3} \cdot$ Andrea De Gottardi ${ }^{2,5} \cdot$ Diego Vergani ${ }^{3}$. \\ Giorgina Mieli-Vergani ${ }^{4}$ \\ 'Epatocentro Ticino, Lugano, Switzerland \\ ${ }^{2}$ Facoltà di Scienze Biomediche, Università della Svizzera Italiana, Lugano, Switzerland \\ ${ }^{3}$ King's College London Faculty of Life Sciences \& Medicine, Institute of Liver Studies, MowatLabs, London, \\ UK \\ ${ }^{4}$ King's College London Faculty of Life Sciences \& Medicine, Paediatric Liver, Gl and Nutrition Centre, \\ MowatLabs, King's College Hospital, London, UK \\ ${ }^{5}$ Ente Ospedaliero Cantonale, Ospedale Italiano, Lugano, Switzerland
}

\title{
Le malattie autoimmuni di fegato nell'adulto
}

aumento delle transaminasi e sierologicamente dalla presenza di autoanticorpi ed alti livelli di immunglobuline G (IgG) [1]. Colpisce tutte le età, i tre quarti dei pazienti sono femmine. La mortalità in assenza di trattamento è elevata, con una sopravvivenza a 5 anni del $50 \%$ e a 10 anni del $10 \%$.

epatite autoimmune (AIH), la colangite biliare primitiva $(\mathrm{PBC})$ e la colangite sclerosante primitiva (PSC) (• ill. 1). La prima colpisce gli epatociti, mentre le seconde colpiscono le vie biliari. Le tre patologie, se non trattate, portano con il tempo a fibrosi e cirrosi epatica, che può portare a insufficienza epatica, tumore e trapianto. Esistono dei pazienti che presentano caratteristiche di AIH associate a caratteristiche di PBC o di PSC, chiamate sindrome di overlap; le patologie si possono presentare contemporaneamente o in tempi diversi. Questo articolo vuole offrire una descrizione clinica delle tre condizioni nel paziente adulto, e non comprende l'entità clinica più recentemente descritta e più rara chiamata colangite a immunoglobuline G4, così come non comprende le sindromi di overlap.

\section{L'epatite autoimmune}

Lepatite autoimmune (AIH) è una malattia infiammatoria del fegato caratterizzata istologicamente da un denso infiltrato mononucleare negli spazi portali che invade il parenchima circostante causando la necrosi degli epatociti (epatite ad interfaccia), biochimicamente da un

\section{Caratteristiche cliniche, sierologiche e biochimiche}

Esistono due forme di AIH, che si distinguono sulla base degli autoanticorpi circolanti. Nel siero dei pazienti con AIH di tipo 1 (AIH-1) si trovano anticorpi contro la muscolatura liscia (anti-smooth muscle antibodies, SMA) e/o contro il nucleo (anti-nuclear antibodies, ANA), mentre l'AIH di tipo 2 (AIH-2) è caratterizzata dalla presenza di anticorpi contro i microsomi di fegato e rene (anti-liver kidney microsomal type 1 antibody, anti-LKM1) e/o contro il citosolo epatico (anti-liver cytosol type 1 antibody, antiLC1).

$\mathrm{AIH}-1$ è di gran lunga più frequente (tre quarti dei casi) e colpisce sia adulti che bambini, mentre AIH-2 è una malattia tipicamente pediatrica/giovanile. Le IgG sono elevate alla presentazione nella maggioranza dei casi, sebbene un 15$25 \%$ di pazienti possano avere valori di IgG normali, soprattutto se la malattia si presenta in modo acuto. Una deficienza parziale di $\operatorname{IgA}$, non associata nella nostra esperienza ad un aumentato ri- schio di infezioni respiratorie, è presente in circa il $40 \%$ dei pazienti con AIH-2.

Agli esami di laboratorio, le transaminasi sono tipicamente molto elevate (10-50 volte la norma), così come spesso la $\gamma$-glutamiltransferasi (GGT), mentre la fosfatasi alcalina è generalmente normale. La bilirubina diretta può essere sia normale che elevata nei casi con presentazione acuta o tardiva. Le IgG sono elevate anche in assenza di cirrosi.

L'AIH dovrebbe essere sospettata ed esclusa in tutti i pazienti con sintomi e segni di malattia epatica non attribuibili ad altre cause note. Vi sono vari modi di presentazione della malattia: la maggioranza degli adulti si presenta con sintomatologia lieve e aspecifica, come stanchezza progressiva, dolori articolari, amenorrea, perdita di peso o dolori addominali. Circa un terzo degli adulti si presenta invece con un'epatite acuta caratterizzata da ittero, malessere, nausea/vomito e/o dolori articolari, simile pertanto ad un'epatite virale acuta. Raramente, l'AIH si manifesta nell'adulto con insufficienza epatica fulminante (encefalopatia epatica di grado II - IV che si sviluppa tra 2 settimane e 2 mesi dopo l'inizio dei sintomi) oppure con complicanze cirrotiche quali ascite, emorragia da varici esofagee, ittero o encefalopatia [1].

Il decorso spontaneo della $\mathrm{AIH}$ può essere fluttuante, con riacutizzazioni e remissioni, un comportamento che può ritardare la diagnosi e l'inizio della tera- 


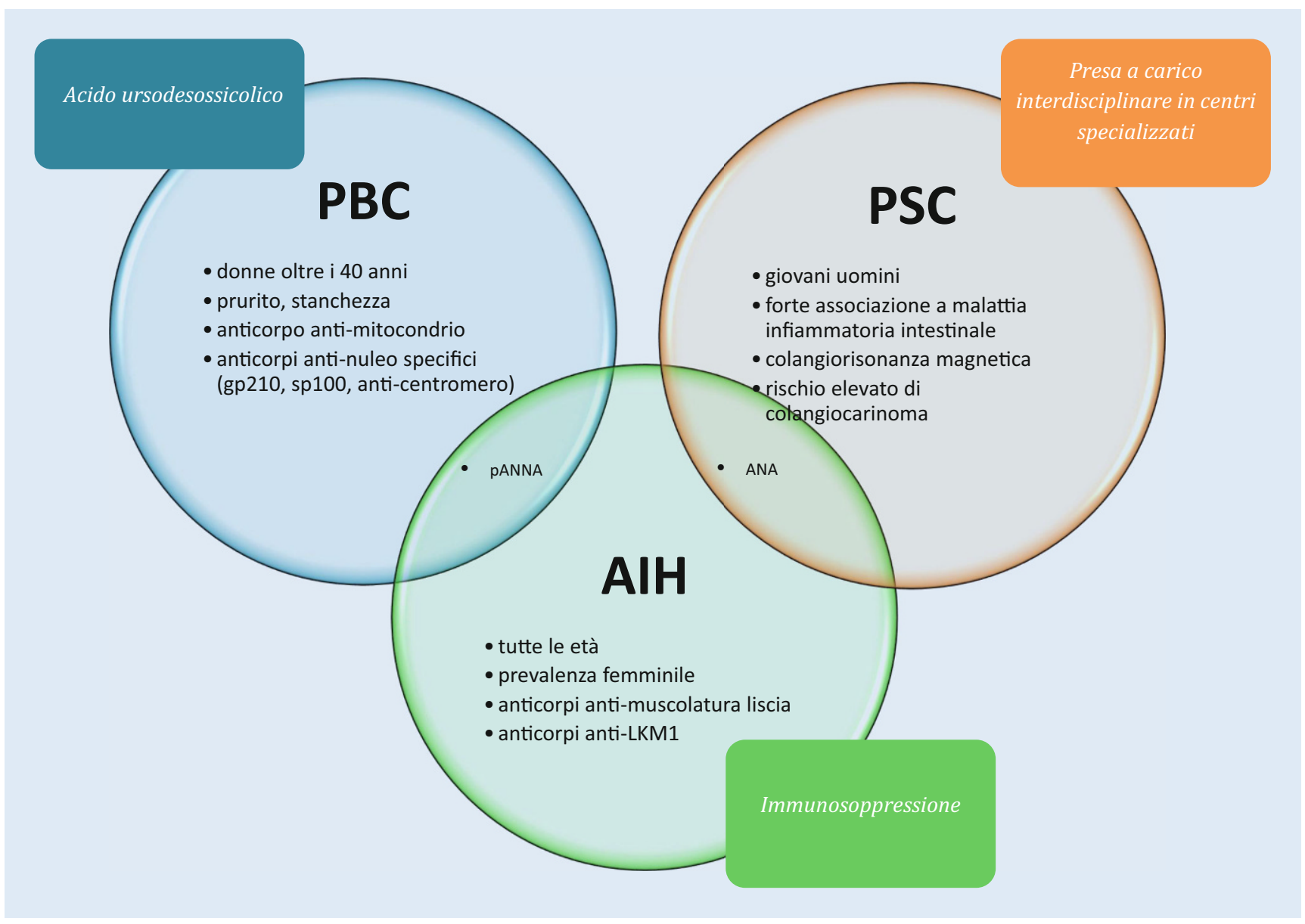

III. 1 A Principali caratteristiche cliniche e sierologiche delle malattie autoimmuni di fegato. AlH epatite autoimmune; $P B C$ colangite biliare primitiva; PSC colangite sclerosante primitiva; $A N A$ anticorpo anti-nucleo; $P A N C A$ anticorpo anti-citoplasma del neutrofilo con pattern perinucleare

pia. Un terzo dei pazienti ha una cirrosi istologica al momento della diagnosi, che può regredire con la terapia, e non è associata a prognosi peggiore [2].

\section{Epidemiologia e fattori di rischio}

LAIH è presente in tutto il mondo. E' considerata una malattia rara, mala prevalenza e l'incidenza riportata in studi recenti è in aumento rispetto a studi più vecchi. La prevalenza annuale attualmente stimata è 20-30/100'000 abitanti, e l'incidenza varia da 1.1 a 3.5/100'000 abitanti.

I dati epidemiologici rappresentano probabilmente una sottostima della reale frequenza della patologia, dato che l'AIH può rimanere non diagnosticata per anni ed essere classificata come 'cirrosi criptogenetica' quando il paziente si presenta con scompenso epatico cronico.
E' riportata una predisposizione genetica associata soprattutto a geni localizzati nella regione dello 'human leukocyte antigen' (HLA) che codificano per proteine coinvolte nella presentazione di peptidi antigenici ai linfociti $\mathrm{T}$ e all'iniziazione delle risposte immunitarie adattative. Lassociazione più forte è con il locus HLA-DRB1. Gli alleli che codificano le molecole HLA-DR3 (DRB1*0301) e DR4 $\left(\mathrm{DRB} 1^{\star} 0401\right)$ conferiscono suscettibilità a AIH-1 nelle popolazioni Europee e Nord Americane, mentre quelli che codificano DR4 conferiscono suscettibilità nelle popolazioni orientali. E' interessante notare che nell'AIH-1 giovanile DR4 ha un effetto protettivo, mentre DR3 conferisce suscettibilità come negli adulti.

La predisposizione genetica a $\mathrm{AIH}-2$ è stata collegata ad alleli che codificano
$\mathrm{DRB}^{\star} 0301$ e $\mathrm{DRB} 1^{\star} 0701$ in Gran Bretagna e Brasile.

La predisposizione genetica all'autoimmunità è testimoniata dal fatto che il $20 \%$ dei pazienti con $\mathrm{AIH}$ ha una concomitante malattia autoimmune extraepatica, e che il $40 \%$ dei pazienti ha una famigliarità per malattie autoimmuni.

Oltre alla predisposizione genetica, sono stati riportati fattori di rischio ambientali quali esposizione a virus (in particolare epatite C), e farmaci quali la nitrofurantoina, la minociclina, le statine, anti-TNF a e rimedi fitoterapici. L'AIH scatenata da farmaci può avere un decorso differente rispetto all'AIH classica, in quanto la terapia immunosoppressiva può spesso venire interrotta senza che il paziente presenti delle recidive [3]. Un'anamnesi farmacologica accurata è pertanto essenziale nei pazienti con un 
Riassunto · Zusammenfassung · Résumé

Schweiz. Gastroenterol. 2021 ·2:56-66 https://doi.org/10.1007/s43472-021-00040-4

(c) Der/die Autor(en) 2021

\section{B. Terziroli Beretta-Piccoli · A. De Gottardi · D. Vergani · G. Mieli-Vergani}

\section{Le malattie autoimmuni di fegato nell'adulto}

\section{Riassunto}

L'epatite autoimmune è una infiammazione cronica del fegato che colpisce tutte le età, caratterizzata da transaminasi e immunoglobuline $\mathrm{G}$ elevate, presenza di autoanticorpi, epatite dell'interfaccia alla biopsia epatica, e ottima risposta alla terapia con steroidi. Se non trattata, ha una sopravvivenza a 5 anni del $50 \%$.

La colangite biliare primitiva è una patologia cronica colestatica autoimmune del fegato che colpisce i medi e piccoli dotti biliari, caratterizzata da preponderanza femminile, e positività dell'anticorpo anti-mitocondrio. La sopravvivenza media della malattia non trattata è 9-10 anni. La terapia di scelta è l'acido ursodesossicolico, che ha un forte impatto sulla storia naturale della malattia. La colangite sclerosante primitiva è la più rara e la più grave delle malattie autoimmuni di fegato. Si caratterizza da forte associazione alle malattie infiammatorie intestinali. I pazienti con PSC hanno un rischio elevato di colangiocarcinoma. Non esistono terapie medicamentose efficaci, e la malattia richiede una presa a carico specialistica multidisciplinare.

Questo articolo offre una panoramica per il clinico delle tre patologie nell'adulto.

Parole chiave

Epatite autoimmune $\cdot$ Colangite biliare primitiva . Colangite sclerosante primitiva . Corticosteroidi · Acido ursodesossicolico

\section{Autoimmunerkrankungen der Leber bei Erwachsenen}

\section{Zusammenfassung}

Die Autoimmunhepatitis ist eine chronische Entzündung der Leber, die in jedem Lebensalter auftreten kann. Bei dieser Erkrankung werden erhöhte Transaminasen und Immunglobulin G-Werte, das Vorhandensein von Autoantikörpern und Grenzzonenhepatitis (Interface-Hepatitis) bei Leberbiopsie verzeichnet. Die Therapie mit Steroiden zeigt sehr gute Ergebnisse. Wird die Krankheit nicht behandelt, liegt die Fünfjahresüberlebensrate bei $50 \%$.

Die primär biliäre Cholangitis (PBC) ist eine

chronische cholestatische Autoimmunerkran- kung der Leber, welche die mittleren und kleinen Gallengänge betrifft und vorwiegend bei Frauen auftritt. Bei dieser Krankheit können antimitochondriale Antikörper nachgewiesen werden. Bei unbehandelter Erkrankung liegt die Lebenserwartung bei 9-10 Jahren. Die Standardtherapie erfolgt mit Ursodesoxycholsäure, die sich gut auf den Krankheitsverlauf auswirkt.

Die primär sklerosierende Cholangitis (PSC) ist die am seltensten auftretende und schwerste Autoimmunerkrankung der Leber. Die Krankheit tritt häufig in Zusammenhang mit entzündlichen Darmerkrankungen auf. PSC-Patienten haben ein erhöhtes Risiko, an einem Cholangiokarzinom zu erkranken. Es gibt keine wirksamen medikamentösen Behandlungen. Die Erkrankung erfordert eine multidisziplinäre fachärztliche Betreuung. Dieser Artikel bietet einen Überblick über die drei Krankheiten bei Erwachsenen.

\section{Schlüsselwörter}

Autoimmune Hepatitis · Primär biliäre Cholangitis (PBC) · Primär sklerosierende Cholangitis (PSC) · Kortikosteroide · Ursodesoxycholsäure

\section{Maladies auto-immunes du foie chez l'adulte}

\section{Résumé}

L'hépatite auto-immune est une inflammation chronique du foie affectant tous les âges, et caractérisée par des taux accrus de transaminases et d'immunoglobulines $\mathrm{G}$, la présence d'auto-anticorps, une hépatite d'interface détectable par une biopsie du foie, et une excellente réponse à la corticothérapie. Si elle n'est pas traitée, le taux de survie à cinq ans est de $50 \%$

La cholangite biliaire primitive (CBP) est une maladie auto-immune chronique cholestatique du foie affectant les voies biliaires de moyen et de petit calibre, caractérisée par une prépondérance féminine et une positivite aux anticorps anti-mitochondriaux. La survie médiane d'une maladie non traitée est de 9 à 10 ans. Le traitement de choix est l'acide ursodésoxycholique qui a un fort impact sur I'histoire naturelle de la maladie. La cholangite sclérosante primitive (CSP) est la plus rare et la plus sévère des maladies auto-immunes du foie. Elle se caractérise par une forte association avec une maladie inflammatoire de l'intestin. Les patients atteints de CSP ont un risque accru de développer un cholangiocarcinome. Il n'existe pas de traitement médicamenteux efficace et la maladie exige une prise en charge multidisciplinaire par des spécialistes. Cet article donne au clinicien un aperçu de ces trois maladies chez l'adulte.

Mots clés

Hépatite auto-immune . Cholangite biliaire primitive (CBP) - Cholangite sclérosante primitive $(\mathrm{CSP}) \cdot$ Corticostéroïdes · Acide ursodésoxycholique sospetto di AIH, e deve includere l'uso di fitoterapici.

\section{Diagnosi}

Non esiste un test diagnostico unico per l'AIH, ma la diagnosi si basa su una combinazione di indicatori clinici (vedi sopra), biochimici (aumento delle transa- minasi), immunologici (aumento delle IgG e positività per autoanticorpi) ed istologici (vedi sotto). E' inoltre essenziale escludere altre cause di malattia epatica che possono avere caratteristiche in comune con l'AIH sia cliniche che sie- 


\begin{tabular}{|c|c|}
\hline $\begin{array}{l}\text { Auto- } \\
\text { anti- } \\
\text { corpo }\end{array}$ & Antigeni target \\
\hline ANA & $\begin{array}{l}\text { Pattern omogeneo, nucleolare e pun- } \\
\text { teggiato: cromatina, istoni, ciclin A, } \\
\text { ribonucleoproteine, DNA elica doppia/ } \\
\text { singola, SSA, SSB, Scl70, Smithr } \\
\text { Pattern rim-like: gp210, nucleoporina } \\
\text { p62, recettore della lamina B } \\
\text { Pattern multiple nuclear dots: Sp100, } \\
\text { proteina della leucemia promielocitica, } \\
\text { Sp140, small ubiquitin-related modifiers } \\
\text { Pattern centromerico: CENP-A, CENP-B, } \\
\text { CENP-C, CENP-D, CENP-E, CENP-F }\end{array}$ \\
\hline
\end{tabular}

SMA Actina filamentosa

Vimentina

Desmina

Anti- Actina

actina

Anti-

CYP2D6

LKM1

Anti- Formiminotransferasi ciclodeaminasi LC1

Anti- $\quad$ O-Phosphoseryl-tRNA (Sec) selenium SLA transferase

pANNA Beta-tubulina isotipo 5; HMG1; HMG2; altri autoantigeni sconosciuti

AMA Sottounità E2 subunit del complesso della piruvato deidrogenasi

\section{Metodi Fre- \\ di \\ quenza \\ dete- \\ Altre malat- Commenti \\ tie di fegato \\ associate}

zione

IIF

AlH-1 e AlH: concomitante pre-

ASC: $75 \%$

PBC: 10

senza di SMA ha specificità

diagnostica del $99 \%$

$65 \% \quad$ PBC: Pattern rim-like e

PSC:

$8-77 \%$

multiple nuclear dots

diagnostici in presenza di

colestasi;

Anti-centromero può

essere associato a PBC

PSC: eventualmente suggestivo di overlap AlH

IIF

AlH-1:

$85 \%$

Pattern VG e VGT IIF speci-

fici per AlH-1

ASC: $75 \%$ Presenza concomitante di

PSC: fino SMA: specificità diagnosti-

a $83 \%$

ca del $99 \%$

PBC: sco-

nosciuto

Test

AlH-1:

Specificità elevata per

mole-

$60 \%$

AlH-1 se ad alto titolo

colari

\section{IIF}

AlH-2:

Test

fino al

Specifico per AlH-2 in

mole-

$90 \%$

assenza di epatite $C$

colari

IIF

Test

AlH-2:

Specifico per AlH-2 in

mole-

colari

$60 \%$

assenza di epatite $C$

Test

mole-

AlH-1 e

Altamente specifico per

AlH-2: 20- AlH

colari $\quad 30 \%$

IIF

AlH-1: 40- Specifico per AlH-1, PSC

$96 \%$ e malattia infiammatoria

PSC: 26

intestinale

$94 \%$

IIF

Test

mole-

colari
PBC: $95 \%$ Altamente specifico per PBC fegato grasso
Epatite virale AlH: pattern omogeneo

Danno epatico nel $75 \%$ dei casi

da farmaci

Malattia da

fegato grasso

Morbo di

Wilson

Carcinoma

epatocellulare

pattern punteggiato 0

nucleolare nel $\sim 25 \%$ dei

casi

PBC: pattern rim-like asso-

ciato a prognosi peggiore

Anti-centromero associato

a ipertensione portale

Danno epatico da farmaci

Pattern V nel 20\% di pazienti AlH-1;

Morbo di

Wilson

Epatite virale

Malattia da

Malattia da

fegato grasso

Epatite virale

Morbo di

Wilson

titolo correla con attività

clinica di malattia

Danno epatico

da farmaci

Epatite C

Titolo correla con attività

di malattia

Ricomparsa post-trapianto predice recidiva di $\mathrm{AlH}-2$;

Anti-LKM1 atipico associa-

to a de novo AlH

Epatite C

Unico autoanticorpo posi-

tivo in $10-30 \%$ dei casi di

AlH-2

Titolo correla con attività

di malattia

Molto ra-

ramente in

epatite $\mathrm{C}$ cro-

nica

\section{Associato a prognosi peg-} giore

-

Raramente è il solo autoanticorpo positivo in AlH-1;

Assente in AlH-2;

suggerisce concomitante

PSC e malattia infiamma-

toria intestinale in pazienti

AlH-1 pANNA positivi

$\mathrm{PBC} / \mathrm{AlH}$ over- Alto rischio di sviluppare lap $\quad$ PBC in pazienti AMA-

Transitoria- positivi senza malattia

mente positi- epatica;

ve nell'insuffi- Può essere associato

cienza epatica a danno dei dotti biliari

acuta istologico anche in assenza di colestasi biochimica

ANA anticorpo anti-nucleo; SMA anticorpo anti-muscolatura liscia; IIF immunofluorescenza indiretta; $V$ vaso; $G$ glomerulo; $T$ tubulo; $A$ IH epatite autoimmune; PSC colangite sclerosante primitiva; $P B C$ colangite biliare primitiva; $L K M$ liver kidney microsomal; $L C 1$ liver cytosol type 1; SLA soluble liver antigen; pANNA perinuclear anti-neutrophil nuclear antibody; HMG1 high mobility group non-histone chromosomal protein; $A M A$ anticorpo anti-mitocondriale 
Tab. 2 Definizioni di risposta insufficiente a terapia con acido ursodeossicolico nella colangite biliare primitiva. Modificato da [7]

\begin{tabular}{|c|c|c|}
\hline Nome dello score & Mesi di terapia con UDCA & Definizione di risposta biochimica \\
\hline Rochester & 6 & ALP $<2 x$ ULN o score Mayo $<4.5$ \\
\hline Barcelona & 12 & ALP diminuita di almeno $40 \%$ e $<$ ULN \\
\hline Parigi l & 12 & $\begin{array}{l}\text { ALP }<3 \times \text { ULN o AST }<2 x \text { ULN o bilirubina } \\
\leq 17 \mu \mathrm{mol} / \mathrm{l}\end{array}$ \\
\hline Rotterdam & 12 & Bilirubina $<$ ULN e/o albumina $\geq$ ULN \\
\hline Toronto & 24 & ALP $<1.67$ ULN \\
\hline Parigi II & 12 & $\begin{array}{l}\text { ALP }<1.5 \text { ULN o AST }<1.5 \text { ULN o bilirubina } \\
\leq 17 \mu \mathrm{mol} / \mathrm{l}\end{array}$ \\
\hline Ehime & 6 & Diminuzione GGT > 70 \% e GGT < ULN \\
\hline UK-PBC & 12 & $\begin{array}{l}\text { Score calcolato con valore di albumina e } \\
\text { piastrine prima di UDCA, e bilirubina, ALP e } \\
\text { AST dopo } 12 \text { mesi di terapia con UDCA }\end{array}$ \\
\hline GLOBE score & 12 & $\begin{array}{l}\text { Score calcolato con età alla diagnosi, e } \\
\text { bilirubina, ALP, albumina e piastrine dopo } \\
12 \text { mesi di terapia con UDCA }\end{array}$ \\
\hline
\end{tabular}

rologiche che istologiche, per esempio le epatiti da virus B, C ed E, il morbo di Wilson, la malattia da fegato grasso e il danno epatico da farmaci.

Istologia - La biopsia epatica è indispensabile per una diagnosi corretta. Il quadro istologico tipico comprende un denso infiltrato mononucleare e plasmacellulare negli spazi portali, che entra nel lobulo epatico includendo gli epatociti situati alla sua periferia (epatite dell'interfaccia).

Le plasmacellule sono di solito presenti in abbondanza all'interfaccia con il lobulo e nel lobulo stesso, ma una loro presenza in basso numero non esclude la diagnosi di AIH. Quando l'AIH si presenta acutamente o ricade, spesso si osserva una epatite panlobulare con collasso del tessuto connettivo dovuto alla necrosi degli epatociti, che si estende dagli spazi portali all'interno del lobulo. La biopsia epatica permette inoltre di valutare il grado di fibrosi ed aiuta ad identificare possibili malattie da overlap o la presenza di epatopatie concomitanti, come la malattia da fegato grasso, che è estremamente frequente nella popolazione occidentale.

Autoanticorpi - La presenza di autoanticorpi circolanti è un criterio diagnostico essenziale. Il $95 \%$ dei pazienti con AIH è positivo per almeno uno degli autoanticorpi tipici della malattia, a condizione che siano stati testati tutti e in ac- cordo con le raccomandazioni pubblicate nel 2004 dal International Autoimmune Hepatitis Group (IAIHG) [4]. La • tab. 1 riassume la rilevanza clinica di ciascuno degli autoanticorpi di pertinenza epatica e gli antigeni da essi riconosciuti. ANA, SMA, anti-LKM-1 e anti-LC-1 dovrebbero essere testati con immunofluorescenza indiretta usando come substrato una triade di tessuti di roditore (stomaco, fegato e rene), che permette di evidenziare tutti gli autoanticorpi tipici dell'AIH. Per anti-LKM-1 e per anti-LC1, di cui si conosce il target antigenico, è anche possibile usare test commerciali, mentre per ANA e SMA, i cui antigeni sono vari e solo in parte conosciuti, le tecniche molecolari al momento non sono completamente affidabili. Lo stesso vale per l'autoanticorpo anti-citoplasma del neutrofilo (ANCA) atipico, che è associato all'AIH-1, alle malattie infiammatorie intestinali e alla colangite sclerosante primitiva e autoimmune (v. sotto). L'immunofluorescenza è una tecnica basata sull'osservazione al microscopio ed è pertanto non standardizzata e dipendente dall'osservatore: questo spiega le differenze fra diversi laboratori frequentemente incontrate nella pratica clinica. Per gli adulti titoli di immunofluorescenza indiretta di 1:40 o più sono considerati positivi. E' importante notare che la positività degli autoanticorpi da sola non è sufficiente per la diagnosi di $\mathrm{AIH}$, dato che gli stessi autoanticorpi, di solito a basso titolo, possono essere presenti in altre malattie di fegato come epatiti virali, morbo di Wilson, danno epatico da farmaci o malattia da fegato grasso. Gli ANA, ANCA e SMA possono essere positivi anche in patologie extraepatiche quali ad esempio il Lupus erythematosus, le vasculiti o la celiachia, rispettivamente. Il solo autoanticorpo specifico per AIH è l'anti-soluble liver antigen (anti-SLA), che non è visibile in immunofluorescen$\mathrm{za}$ indiretta e deve essere testato con test molecolari nei pazienti con sospetta $\mathrm{AIH}$.

\section{Terapia}

Siccome l'AIH non trattata progredisce verso la cirrosi, la terapia va iniziata al più presto e ha lo scopo di spegnere l'infiammazione epatica, evitando l'evoluzione fibrotica, e di migliorare $\mathrm{i}$ sintomi clinici. La remissione biochimica nei pazienti adulti viene definita come la normalizzazione delle transaminasi e delle IgG, mentre per i bambini si richiede in aggiunta la negativizzazione degli autoanticorpi $[1,5]$. La remissione biochimica correla con l'assenza di infiammazione a livello istologico. Il trattamento si basa sui corticosteroidi, con o senza l'aggiunta di azatioprina, ed è altamente efficace, ma $\sim 80 \%$ dei pazienti lo deve continuare per tutta la vita, con il rischio di effetti collaterali. I pazienti che non vanno in remissione, o perche' non rispondono adeguatamente alla terapia immunosoppressiva o perchè non la tollerano, vanno incontro ad una progressione del danno epatico e dunque potenzialmente al trapianto di fegato. Farmaci alternativi sono usati in questi casi in centri specializzati, ma nessuno di questi farmaci è stato ufficialmente approvato per la terapia dell'AIH.

\section{Trattamento iniziale e di secondo livello}

Glucocorticoidi Il predniso(lo)ne e' lo steroide di scelta per il trattamento dell'AIH. Il trattamento deve essere adattato a ciascun singolo paziente, a seconda della gravità della malattia, dell'età, delle comorbidità e della risposta: occorre pertanto adattare le tabelle proposte dalle guide- 
lines al singolo paziente. Negli adulti le guidelines dell'American Association for the Study of Liver Diseases (AASLD) del 2019 suggeriscono di cominciare con una dose di predniso(lo)ne 60mg/die se la malattia è severa (ittero, coagulopatia) e di $20-40 \mathrm{mg} /$ die se la malattia non è severa; le guidelines dell'European Society for the Study of the Liver (EASL) del 2015 indicano una dose iniziale di $0.5-1 \mathrm{mg} / \mathrm{Kg}$ al giorno. Entrambe le guidelines suggeriscono di aggiungere azatioprina (50$150 \mathrm{mg} / \mathrm{die}$ ), che ha un effetto di 'steroid sparing' (risparmio di steroidi), ma puo' essere epatotossica in pazienti cirrotici e/o itterici, solo dopo circa due settimane di monoterapia steroidea, quando si e' raggiunto un parziale controllo della malattia.

Per evitare gli effetti collaterali del cortisone (Cushing, diabete, osteoporosi, cataratta, psicosi, ipertensione), che sono correlati soprattutto alla dose iniziale alta e che sono reversibili, la dose di predniso(lo)ne deve essere diminuita rapidamente in base ai livelli delle transaminasi misurati settimanalmente, dato che la emivita di questi enzimi e' breve (1747 ore), in contrasto alla emivita delle IgG (21-28 giorni). Se durante la diminuzione della dose degli steroidi le transaminasi smettono di scendere o tendono ad aumentare, viene aggiunta l'azatioprina. Dato che l'AIH è molto sensibile agli steroidi, di solito una dose di mantenimento di prednisolone di $5 \mathrm{mg} /$ die, con o senza azatioprina secondo il bisogno, è sufficiente a mantenere il controllo della malattia.

Budesonide: il budesonide è uno steroide con un potente effetto topico (utilizzato anche in forma inalatoria per trattare le infiammazioni delle mucose respiratorie) ed un alto primo passaggio (firstpass) a livello epatico (> $90 \%)$, rendendolo una terapia attraente per AIH. Tuttavia non può essere usato in pazienti cirrotici, in cui è associato a seri effetti collaterali. In uno studio multicentrico Europeo il trattamento con budesonide ( $3 \mathrm{mg}$ tre volte al giorno) ed azatioprina $(1-2 \mathrm{mg} / \mathrm{Kg} /$ die $)$ è stato paragonato al trattamento con prednisone $(40 \mathrm{mg} / \mathrm{die})$ ed azatioprina (1-2 mg/Kg/die). Lo studio ha mostrato una percentuale maggiore di pazienti che raggiungevano transamina- si normali senza effetti collaterali degli steroidi nella coorte trattata con budesonide, portando alla licenza di questo farmaco come trattamento iniziale della AIH. Tuttavia, nello studio la percentuale di remissione con budesonide ed azatioprina è nettamente inferiore a quella che si ottiene con monoterapia di predniso(lo)ne a cui l'azatioprina viene aggiunta più tardi. Il budesonide perciò, per il momento, non e' un farmaco di prima scelta per il trattamento iniziale dell'AIH, ma può essere una valida alternativa per il mantenimento della remissione a lungo termine in pazienti non cirrotici che rispondono alla terapia standard, in particolare in presenza di effetti collaterali da steroidi.

\section{Inibitori della sintesi delle purine Gli in-} ibitori della sintesi delle purine sono dei potenti agenti 'steroid sparing' e vengono ampiamente usati nell'AIH.

Azatioprina: Il più comunemente usato inibitore della sintesi delle purine è l'azatioprina, che è bene iniziare solo quando si è raggiunta una parziale remissione con la terapia steroidea, perché l'azatioprina può essere epatotossica in pazienti cirrotici e/o itterici. Si comincia di solito con una dose bassa $(50 \mathrm{mg} / \mathrm{die}$ nell'adulto) per evitare possibili effetti collaterali (mielotossicità, epatossicità) che viene a poco a poco incrementata, dopo controlli ematologici, fino alla dose di mantenimento (1-2 mg/kg/die). I pazienti possono accusare una modesta nausea, che di solito migliora col tempo e se il farmaco viene assunto col cibo e/o la dose viene divisa in due somministrazioni giornaliere. La mielotossicità da azatioprina è dose-dipendente ed è influenzata dalla variabilità individuale della farmacocinetica dell'azatioprina. Lazatioprina sì può usare in gravidanza senza effetti collaterali sul feto. Si possono fare test genetici per identificare mutazioni dellenzima tiopurina S-metiltransferasi (TPMT) che predispongono a grave tossicità midollare, ma anche pazienti senza mutazioni TPMT possono sviluppare mielotossicità, mentre alcuni portatori della mutazione tollerano il farmaco senza problemi. Data la variabilità del metabolismo dell'azatioprina, può essere utile misurare i livelli sierici dei suoi metaboliti attivi (6-thioguanine nucleotides, 6-TGN) per monitorare sia l'efficacia che l'aderenza al trattamento, e del suo metabolita tossico (6-methylthioinosine 5'monophosphate, 6-MMP) per monitorare la tossicità. In pazienti che metabolizzano l'azatioprina con una preferenziale produzione di metaboliti tossici, viene consigliata l'aggiunta di allopurinolo, che dirige il metabolismo della tiopurina verso i metaboliti attivi non tossici. Una volta raggiunta la remissione, è possibile mantenerla con azatioprina in monoterapia, ma i migliori risultati vengono ottenuti con una combinazione individuale delle più basse dosi di predniso(lo)ne ed azatioprina che mantengono livelli di transaminasi e IgG normali nel singolo paziente.

Micofenolato mofetile: Il micofenolato mofetile (MMF) è un inibitore della sintesi delle purine, utilizzato per prevenire il rigetto nei pazienti sottoposti a trapianto di organo. Dato il suo basso profilo tossico, MMF è il trattamento di scelta, in associazione con predniso(lo)ne, in pazienti con AIH che non tollerano o che non rispondono all'azatioprina, con buoni risultati soprattutto nei primi. MMF è teratogenico e va usato con cautela nelle donne in età fertile.

\section{Trattamenti di terzo livello}

Il $10-15 \%$ dei pazienti non raggiunge la remissione biochimica con il trattamento standard, e necessitano di trattamenti di terzo livello. Farmaci che sono stati usati a questo scopo comprendono inibitori della calcineurina (ciclosporina e tacrolimus), rapamicina, ed anticorpi monoclonali anti-linfociti B (rituximab) o antiTNFa. Lesperienza e' limitata a pochi casi con risultati variabili e la terapia può solo essere condotta in centri specializzati, anche per la possibilità di seri effetti collaterali.

\section{Trapianto epatico}

Il 2-3\% dei trapianti epatici in Europa e circa il $5 \%$ negli Sati Uniti viene fatto per AIH. Il trapianto epatico è indicato quando l'AIH si presenta come epatite fulminante non responsiva a terapia steroidea entro 5 giorni, o quando progredisce ad epatopatia cronica scompensata malgrado la terapia. La sopravvivenza 
dopo trapianto a 5 anni è ottima (80$90 \%$ ), ma recidiva di $\mathrm{AIH}$ è riportata in circa il $20 \%$ dei casi e deve essere trattata con il trattamento standard per AIH. La ricorrenza può avvenire anche anni dopo il trapianto e per questo si consiglia di continuare il trattamento steroideo a basse dosi, insieme al trattamento antirigetto, indefinitamente in pazienti trapiantati per AIH.

\section{La Colangite Biliare Primitiva}

La PBC è una patologia cronica colestatica autoimmune del fegato che colpisce i medi e piccoli dotti biliari, caratterizzata da preponderanza femminile, e positività dell'anticorpo anti-mitocondrio (AMA) [6]. Linfiammazione dei dotti biliari porta alla loro distruzione, con conseguente cirrosi, ipertensione portale e insufficienza epatica. L'attacco autoimmune è diretto contro un antigene ben definito, la sottounità E2 del complesso proteico denominato piruvato deidrogenasi mitocondriale (PDC-E2). Il tipico paziente è una donna oltre i 40 anni.

\section{Caratteristiche cliniche e biochimiche}

La PBC comprende un largo spettro di manifestazioni cliniche, che vanno dalla lieve colestasi asintomatica al prurito colestatico severo alle complicanze cirrotiche. Si tratta pertanto di una malattia eterogenea, e il rischio di progressione della malattia nel singolo paziente può essere predetto da vari scores (•tab. 2), il cui uso è raccomandato nella pratica clinica al fine di individuare pazienti da avviare per tempo verso terapie di seconda linea, terapie sperimentali o eventualmente verso il trapianto epatico. Gli scores si basano sulla risposta alla terapia con UDCA e sono pertanto diversi dallo stadio di malattia alla diagnosi, che rappresenta di per sé un fattore di rischio per outcome peggiore.

Oltre la metà dei pazienti con $\mathrm{PBC}$ sono sintomatici per prurito e/o stanchezza ("fatigue"), sintomi che hanno un importante impatto sulla qualità di vita [7]. Altri sintomi frequenti sono xeroftalmia e xerostomia, senza necessariamente che i criteri diagnostici della sindrome di
Sjögren siano soddisfatti, e il fenomeno di Raynaud, che spesso è isolato, ma l'8 \% dei pazienti con PBC hanno anche una sclerodermia a forma limitata.

\section{Epidemiologia e fattori di rischio}

Benché la PBC soddisfi la definizione di malattia rara (prevalenza $<50 / 10$ '000 in tutte le popolazioni studiate), si stima che 1 donna su 1000 di età superiore ai 40 anni sia affetta da PBC [7]. La prevalenza femminile, condivisa con la maggioranza delle malattie autoimmuni, è particolarmente spiccata nella $\mathrm{PBC}$, dove 9 pazienti su 10 sono donne [8]. La malattia non esiste in pediatria, il caso più giovane riportato in letteratura è quello di una ragazza di 15 anni [8].

Il rischio di $\mathrm{PBC}$ è conferito sia da fattori genetici, di cui l'alta concordanza fra gemelli omozigoti (63\%) è una chiara testimonianza, sia da fattori ambientali, quali il fumo, infezioni urinarie recidivanti e esposizione a xenobiotici come il 2-octyonic acid contenuto in cosmetici e prodotti alimentari. Lassociazione con infezioni urinarie recidivanti è probabilmente dovuta a sequenze aminoacidiche conservate fra l'Escherichia coli e la proteina PDC-E2.

La predisposizione genetica è conferita soprattutto da varianti del gene dell'HLA, ma anche di altri geni implicati nel sistema immunitario.

\section{Diagnosi}

La diagnosi di PBC è semplice, ed è posta nel caso in cui almeno due dei seguenti tre criteri sono soddisfatti:

- Rialzo inspiegato per almeno 6 mesi della fosfatasi alcalina (ALP) di origine epatica

- Presenza nel siero degli anticorpi anti-mitocondrio (AMA) ad un titolo di almeno 1:40

- Tipico danno dei dotti biliari all'esame istologico del tessuto epatico

Una dilatazione delle vie biliari deve essere esclusa, spesso una semplice ecografia addominale è sufficiente.

L'AMA è estremamente specifico per la PBC, essendo consideratol'autoanticorpo più specifico nelle patologie umane [9].
Hanno la stessa specificità, e pertanto lo stesso valore diagnostico, anche due sottotipi dell'ANA, cioè quelli che all'immunofluorescenza su cellule HEp-2 danno un pattern nucleare di tipo "multiple dots" o "rim-like", corrispondente molto spesso all'anti-sp100 e all'antigp210 se testati con test molecolari (questi anticorpi vengono anche chiamati ANA PBC-specifici). È pertanto di estrema rilevanza diagnostica che il laboratorio riporti il pattern di immunofluorescenza, e che il clinico ne conosca il significato diagnostico. L'ANA rim-like è associato ad un decorso più severo della PBC. Gli ANA PBC-specifici sono presenti in circa un terzo dei pazienti con $\mathrm{PBC}$, e sono estremamente utili nella diagnosi della PBC AMAnegativa.

La biopsia epatica non è indispensabile per la diagnosi, ma è necessario stabilire lo stadio di malattia al momento della diagnosi, cosa che può essere fatta anche con metodi non invasivi, in particolare con l'elastometria epatica, e con la misura della conta piastrinica, della bilirubina e dell'albumina [7]. I pazienti con malattia avanzata alla diagnosi hanno una sopravvivenza diminuita, e andrebbero pertanto indirizzati ad un centro di riferimento terziario.

Lalterazione istologica tipica della $\mathrm{PBC}$ è la colangite cronica non suppurativa e distruttiva, cioè un'infiltrazione linfocitica dellepitelio dei dotti biliari che conduce alla dottopenia e alla fibrosi dei campi portali [9]. In assenza di terapia, l'epatite dell'interfaccia è molto frequente, ma questo non deve portare a sovradiagnosticare una coesistente AIH [9].

\section{Terapia}

La sopravvivenza media della PBC non trattata è 9-10 anni [7]. E' pertanto essenziale riconoscere e trattare la malattia prima che progredisca. Infatti, se il trattamento è iniziato in fase precoce, la sopravvivenza diventa simile a quella della popolazione generale [10].

Lo scopo della terapia è quello di ottenere una risposta biochimica completa, la cui definizione fino al recente passato era data da un valore della fosfatasi 
alcalina (ALP) $<1.5$ o 1.67 il valore superiore della norma (ULN) dopo 12 o 24 mesi di terapia con UDCA [7, 8]. Il valore della ALP è un endpoint surrogato della sopravvivenza [7]. Dati più recenti indicano che una completa normalizzazione della ALP e un valore della bilirubina totale $<0.6 \times$ ULN migliorano la sopravvivenza, e dovrebbero pertanto rappresentare l'obiettivo della terapia [11]. E' importante tenere conto che uno stadio avanzato di malattia al momento della diagnosi è un fattore di rischio indipendente per una prognosi peggiore [12], evidenziando l'importanza di una diagnosi precoce.

\section{Acido ursodesossicolico}

Lacido ursodesossicolico (UDCA) è la terapia di prima linea della PBC: la sua efficacia nel diminuire il rischio di morte e di trapianto epatico è dimostrata solidamente da studi clinici e da esperienza nella pratica reale a lungo termine. La dose raccomandata nella $\mathrm{PBC}$ è di 13 $15 \mathrm{mg} / \mathrm{kg}$ di peso corporeo al giorno. Il benefico dell'UDCA è mantenuto indipendentemente dallo stadio di malattia e dalla risposta biochimica: pertanto, il farmaco va mantenuto a vita in tutti i pazienti con $\mathrm{PBC}$, con la rara eccezione dei pazienti che non lo tollerano, spesso per diarrea.

L'UDCA è un acido biliare naturalmente presente nellorganismo umano: viene infatti generato dai batteri nel colon a partire dall'acido chenodesossicolico, ed è il solo acido biliare idrofilico. I meccanismi d'azione sono molteplici e non chiariti completamente; comprendono una diminuzione della quantità di colesterolo nella bile (fatto che spiega il suo impiego nel trattamento dei calcoli biliari), una minore tossicità della bile, un aumento della coleresi, così come meccanismi immunomodulatori.

L'UDCA è un farmaco sicuro, può essere dato anche in gravidanza e durante l'allattamento. Gli effetti collaterali sono rari, e prevalentemente di tipo gastrointestinale (diarrea, dolori addominali).

\section{Acido obeticolico}

Lacido obeticolico (OCA) è un derivato semisintetico dell'acido chenodesossicolico, un acido biliare primario. Ė la sola te- rapia di seconda linea approvata. Lacido obeticolico è un agonista del recettore nucleare farnesoid-X, e agisce principalmente diminuendola sintesi di acidibiliari, e aumentando la perdita di acidi biliari con le feci [6]. L'OCA è indicato nei pazienti intolleranti all'UDCA e in quelli con risposta insufficiente all'UDCA, definita come ALP >1.67 x ULN e/o bilirubina $>2 \times$ ULN dopo 6 mesi di UDCA. La dose iniziale è di $5 \mathrm{mg} /$ die, titrabile dopo $6 \mathrm{mesi}$ a $10 \mathrm{mg} /$ die in caso di risposta insufficiente. È estremamente importante ridurre notevolmente la dose nei pazienti con cirrosi CHILD B e C: la dose iniziale in questi casi è di $5 \mathrm{mg}$ a settimana, incrementabile secondo tolleranza ed effetto fino a $10 \mathrm{mg} 2$ volte a settimana. Sono infatti stati riportati casi di insufficienza epatica grave in pazienti cirrotici con la dose piena di OCA. L'effetto secondario principale è l'esacerbazione del prurito, che è dose-dipendente, e porta alla sospensione del farmaco in 1-10\% dei pazienti. OCA ha anche un impatto sul profilo lipidico, in particolare provoca una diminuzione del colesterolo HDL; l'impatto sul rischio cardiovascolare di questa alterazione non è noto.

Agonisti del FXR di seconda generazione (chiamati non-steroidali), che promettono un miglior profilo di effetti collaterali e maggiore efficacia, sono in fase di studi clinici (tropifexor, cilofexor).

\section{Fibrati}

I fibrati sono usati da decenni per il trattamento della $\mathrm{PBC}$ nei pazienti con risposta incompleta all'UDCA, in combinazione con quest'ultimo. Si tratta di farmaci registrati per il trattamento dell'iperlipidemia, formalmente controindicati nei pazienti epatopatici. Il fenofibrato e il bezafibrato hanno dimostrato un beneficio biochimico (riduzione della ALP) in numerosi studi con un basso numero di pazienti [6]. Nel 2018 sono stati pubblicati da Corpechot e colleghi i risultati di uno studio randomizzato, placebo-controllato di fase 3 comprendente 100 pazienti con $\mathrm{PBC}$ e risposta parziale all'UDCA: 50 sono stati trattati con UDCA e bezafibrato ( $400 \mathrm{mg}$ al giorno) e 50 con UDCA e placebo, per 24 mesi: il $67 \%$ dei pazienti nel braccio bezafibrato hanno normalizzato completamente la ALP, e il $31 \%$ hanno normalizzato tutti i test epatici (endpoint primario dello studio); nel braccio placebo, solo il $2 \%$ dei pazienti ha normalizzato la ALP e nessuno ha raggiunto l'endpoint primario [13]. Un vantaggio importante dei fibrati è il loro beneficio sul prurito [14]. Il beneficio dimostrato dai fibrati nella $\mathrm{PBC}$ ha stimolato lo sviluppo di nuovi e più mirati farmaci agonisti dei PPAR, che sono attualmente in studi clinici anche già di fase 3 (elafibranor, seladelpar).

L'evento avverso più frequente dei fibrati $(10 \%)$ sono mialgie, seguito da aumento delle transaminasi $>5$ x ULN (6\%) e aumento della bilirubina. Un incremento della creatinina è un ben noto effetto collaterale reversibile dei fibrati, che potrebbe però essere dovuto ad una aumentata sintesi di creatinina nei muscoli piuttosto che ad una diminuita funzione renale. Eventuali interazioni farmacologiche vanno accuratamente indagate prima di prescrivere i fibrati; inoltre, il rischio di tossicità delle statine è aumentata se combinate con fibrati. Non ci sono dati sull'uso dei fibrati in pazienti con cirrosi CHILD B e C.

\section{Trapianto epatico}

Pazienti con risposta parziale alla terapia medicamentosa progrediscono verso l'insufficienza epatica e avranno pertanto bisogno di un trapianto, le cui indicazioni nella PBC sono uguali a quelle delle altre forme di epatopatia. Il prurito intrattabile può rappresentare una valida indicazione al trapianto. La PBC recidiva nell'organo trapiantato in almeno il $20 \%$ dei casi, raramente con necessità di un secondo trapianto. L'UDCA e una immunosoppressione con ciclosporina diminuiscono il rischio di recidiva.

\section{Terapia dei sintomi}

I sintomi tipici della $\mathrm{PBC}$ sono il prurito e la stanchezza, che colpiscono oltre la metà dei pazienti. Entrambi i sintomi non rispondono alla terapia standard con UDCA; la stanchezza può persistere anche dopo il trapianto, mentre il prurito incoercibile scompare dopo il trapianto. La terapia farmacologica di prima linea del prurito colestatico, dopo avere esaurito le misure locali come emollienti, è la co- 
lestiramina, che va assunta almeno 2 ore prima o dopo l'UDCA, e che ha una efficacia limitata e spesso è mal tollerata. Terapie di seconda linea sono la rifampicina (150-300 mg/die), che però è epatotossica e può avere interazioni farmacologiche importanti. Gli antistaminici sedativi sono spesso prescritti nel tentativo di alleviare il prurito notturno. Altre possibili terapie medicamentose comprendono il naltrexone e il nalmefene, e gli inibitori del reuptake della serotonina [7].

La stanchezza è molto difficile da trattare, e cause diverse dalla PBC vanno escluse. Gruppi di autoaiuto e modifiche dello stile di vita possono essere di aiuto.

\section{Complicanze}

\section{Osteopenia/osteoporosi}

Losteoporosi è una complicanza frequente della PBC. E' importante evitare altri fattori di rischio per l'osteoporosi, quali il fumo, la malnutrizione, la carenza di vitamina $\mathrm{D}$ e la sedentarietà. Una densitometria ossea al momento della diagnosi e poi regolarmente nel decorso è raccomandata, ma è possibile che non venga rimborsata dalle casse malati in Svizzera. La terapia dell'osteoporosi associata alla PBC non è studiata, e si basa sui dati raccolti nel trattamento dell'osteoporosi post-menopausale.

\section{Carcinoma epatocellulare}

Il rischio di carcinoma epatocellulare nella cirrosi da PBC è di 0,34-0,36\% all’anno, ed è modulato dalla presenza di altri fattori di rischio, quali il sesso maschile, la steatosi epatica, la sindrome metabolica, la famigliarità, l'età e il consumo di alcol. I pazienti con cirrosi da $\mathrm{PBC}$ necessitano di uno screening semestrale con ecografia addominale, analogamente a quanto raccomandato per tutti i pazienti cirrotici [7].

\section{Ipertensione portale}

Malgrado i pazienti con PBC possano sviluppare ipertensione portale ad uno stadio più precoce di malattia rispetto ad epatopatie non colestatiche, le raccomandazioni di Baveno VI per lo screening delle varici esofagee sono valide anche nella PBC, ma non lo sono i criteri estesi di Baveno [15].

\section{La Colangite Sclerosante Primitiva}

La colangite sclerosante primitiva (PSC) è la più rara e la più grave delle malattie autoimmuni di fegato. La sua caratteristica clinica principale è la forte associazione alle malattie infiammatorie intestinali (IBD), che hanno tipicamente un fenotipo differente rispetto a quelle non associate a PSC, coinvolgendo quasi sempre il colon (pancolite, a predominanza destra) con una ileite terminale e risparmio del retto. La PSC provoca un'infiammazione cronica delle vie biliari intra- ed extraepatiche, e la sua patogenesi rimane sconosciuta. I pazienti con PSC hanno un rischio elevato di colangiocarcinoma, e, in caso di concomitante IBD, di carcinoma del colon.

\section{Caratteristiche cliniche e biochimiche}

Tipicamente la malattia si presenta in pazienti maschi fra i 30 e i 40 anni affetti da IBD, che nelle popolazioni occidentali è presente nei due terzi dei pazienti con PSC. Circa l' $8 \%$ dei pazienti con IBD ha una concomitante PSC. Le due patologie possono essere diagnosticate nello stesso momento, o in tempi diversi. È interessante notare che una IBD può insorgere anche dopo trapianto epatico per PSC, e che una PSC può a sua volta insorgere dopo colectomia totale per IBD.

La maggior parte dei pazienti è asintomatico alla diagnosi, soprattutto se diagnosticato in fase precoce di malattia. Malgrado l'impatto della diagnosi sulla sorveglianza per neoplasie, un depistaggio della PSC nei pazienti con IBD non è generalmente raccomandato [16]. Una colestasi biochimica può essere assente, ma occorre tener conto che c'è una naturale importante fluttuazione dei valori colestatici nella PSC. Pertanto, è necessario un alto grado di sospetto di PSC nei pazienti con IBD e epatopatia.

I sintomi più frequenti comprendono prurito, stanchezza, ittero, e dolori addominali. C’è una prevalenza maschile, due terzi dei pazienti sono maschi, anche se è possibile che la malattia sia meno aggressiva e pertanto sottodiagnosticata nelle donne [16].
In pediatria, è definita una entità clinica denominata colangite sclerosante autoimmune, che rappresenta una sovrapposizione di AIH con colangite sclerosante, e colpisce la metà dei bambini con AIH (prevalentemente di tipo 1) [17].

\section{Epidemiologia e fattori di rischio}

La PSC è una malattia rara, la prevalenza riportata è di circa 1 caso per 10 '000 abitanti, e l'incidenza varia fra 0.4 e 2 casi per 100'000 abitanti per anno [16]. Un quarto dei pazienti ha altre malattie autoimmuni concomitanti. Sono stati descritti fattori genetici predisponenti, ma probabilmente giocano un ruolo meno importante rispetto a fattori ambientali [18].

\section{Diagnosi}

La diagnosi di PSC è posta con la colangiorisonanza. La biochimica epatica può, ma non deve, mostrare una colestasi. È importante escludere forme secondarie di colangite sclerosante, come per esempio la colangite a IgG4, la colangite ischemica, la colangite da malattia critica, la colangite piogena, forme genetiche o secondarie a HIV o sarcoidosi. In caso di forte sospetto e colangiorisonanza normale, è raccomandata la biopsia epatica per ricerca di colangite sclerosante dei piccoli dotti, che ha una prognosi più benigna ma può evolvere verso la PSC. Lautoanticorpo ANCA, in particolare il pANCA atipico, è positivo in gran parte dei casi e può supportare la diagnosi (- Tab. 1).

Nei pazienti con nuova diagnosi di PSC senza IBD, è raccomandata una panendoscopia digestiva per il depistaggio della IBD; in seguito la colonoscopia è raccomandata ogni 5 anni, o in presenza di sintomi [19].

\section{Terapia}

La presa a carico della PSC è complessa e richiede un approccio interdisciplinare in un centro di riferimento con esperienza.

Non esistono terapie medicamentose efficaci. L'UDCA ha mostrato risultati variabili, e la sua efficacia in termini di prevenzione della cirrosi e sopravvivenza 
non è documentata. Tuttavia è largamente prescritto, alla dose di $13-15 \mathrm{mg} / \mathrm{kg} /$ giorno, e spesso migliora la colestasi biochimica. Dosi elevate $(28-30 \mathrm{mg} / \mathrm{die})$ sono invece dannose.

Nuove terapie medicamentose sono in fase di studio clinico.

Attualmente la sola terapia efficace è il trapianto epatico. Tuttavia, la malattia ricorre in circa il $20 \%$ dei pazienti, con un impatto sulla sopravvivenza sia dellorgano che del paziente.

\section{Complicanze}

Il $10-20 \%$ dei pazienti con PSC sono colpiti da colangiocarcinoma, di cui la metà dei casi è diagnosticata nell'anno che segue la diagnosi di PSC. In seguito, l'incidenza annuale è dello $0.5-1.5 \%$. Il colangiocarcinoma è una malattia aggressiva, con poche possibilità di terapia, e una sopravvivenza a 5 anni intorno al $20 \%$. Il colangiocarcinoma può essere localizzato anche nella cistifellea, così che ogni polipo colecistico, indipendentemente dalle dimensioni, rappresenta un'indicazione alla colecistectomia nei pazienti con PSC.

Anche il rischio di carcinoma del colon è elevato nei pazienti con PSC e IBD, e giustifica una colonoscopia annuale per il depistaggio. liari.

Un quarto dei pazienti ha calcoli bi-

Le colangiti batteriche recidivanti sono una complicanza frequente e temuta della PSC, e possono indicare il trapianto epatico.

Le stenosi biliari dominanti, dove spesso è difficile distinguere una stenosi maligna da una infiammatoria, sono un'altra frequente e temuta complicanza della PSC, necessitante una terapia endoscopica da parte di gastroenterologi particolarmente esperti.

\section{Conclusione}

Le malattie autoimmuni di fegato sono considerate malattie rare, ma la loro frequenza è in aumento, probabilmente grazie anche ad una maggior consapevolezza della loro esistenza da parte dei clinici e di test diagnostici più performanti. È essenziale che vengano diagnosticate e trattate appropriatamente per tempo, al fine di evitare la progressione verso l'insufficienza epatica. La PSC è complessa, non ha un trattamento medicamentoso efficace, e richiede una presa a carico interdisciplinare in un centro di riferimento.

\section{Indirizzo per la corrispondenza}

Benedetta Terziroli Beretta-Piccoli

Epatocentro Ticino

Lugano, Switzerland

benedetta.terziroli@usi.ch

Funding. Open access funding provided by Università della Svizzera italiana

\section{Direttive etiche}

Conflitto di interessi B. Terziroli Beretta-Piccoli, A. De Gottardi, D. Vergani e Giorgina Mieli-Vergani affermano che non ci sono conflitti di interessi.

Gli autori non hanno condotto alcuno studio su esseri umani o animali per questo articolo. Per gli studi elencati, le linee guida etiche ivi indicate si applicano in ogni caso.

Open Access. Dieser Artikel wird unter der Creative Commons Namensnennung 4.0 International Lizenz veröffentlicht, welche die Nutzung, Vervielfältigung Bearbeitung, Verbreitung und Wiedergabe in jeglichem Medium und Format erlaubt, sofern Sie den/die ursprünglichen Autor(en) und die Quelle ordnungsgemäß nennen, einen Link zur Creative Commons Lizenz beifügen und angeben, ob Änderungen vorgenommen wurden.

Die in diesem Artikel enthaltenen Bilder und sonstiges Drittmaterial unterliegen ebenfalls der genannten Creative Commons Lizenz, sofern sich aus der Abbildungslegende nichts anderes ergibt. Sofern das betreffende Material nicht unter der genannten Creative Commons Lizenz steht und die betreffende Handlung nicht nach gesetzlichen Vorschriften erlaubt ist, ist für die oben aufgeführten Weiterverwendungen des Materials die Einwilligung des jeweiligen Rechteinhabers einzuholen.

Weitere Details zur Lizenz entnehmen Sie bitte der Lizenzinformation auf http://creativecommons.org/ licenses/by/4.0/deed.de.

\section{Bibliografia}

1. Mieli-Vergani G, Vergani D, Czaja AJ, Manns MP, KrawittEL, Vierling JM, Lohse AW, Montano-Loza AJ (2018) Autoimmune hepatitis. Nat Rev Dis Primer 4:18017. https://doi.org/10.1038/nrdp.2018.17

2. Di Giorgio A, Hadzic N, Dhawan A, Deheragoda M, Heneghan MA, Vergani D, Mieli-Vergani G, Samyn M (2020) Seamless management of juvenile autoimmune liver disease: long-term medical and social outcome. J Pediatr 218:121-129.e3. https:// doi.org/10.1016/j.jpeds.2019.11.028
3. European Association for the Study of the Liver (2015) EASL clinical practice guidelines: autoimmune hepatitis. J Hepatol 63:971-1004. https://doi.org/10.1016/j.jhep.2015.06.030

4. Vergani D, Alvarez F, Bianchi FB, Cançado ELR, Mackay IR, Manns MP, Nishioka M, Penner E, International Autoimmune Hepatitis Group (2004) Liver autoimmune serology: a consensus statement from the committee for autoimmune serology of the International Autoimmune Hepatitis Group. J Hepatol 41:677-683. https://doi. org/10.1016/j.jhep.2004.08.002

5. Mieli-Vergani G, Vergani D, Baumann U, Czubkowski P, Debray D, Dezsofi A, Fischler B, Gupte G, Hierro L, Indolfi G, Jahnel J, Smets F, Verkade HJ, Hadzic N (2017) Diagnosis and management of paediatric autoimmune liver disease: ESPGHAN hepatology committee position statement. J Pediatr Gastroenterol Nutr. https://doi.org/10.1097/ MPG.0000000000001801

6. Gulamhusein AF, Hirschfield GM (2020) Primary biliary cholangitis: pathogenesis and therapeutic opportunities. Nat Rev Gastroenterol Hepatol 17:93-110. https://doi.org/10.1038/s41575-0190226-7

7. European Association for the Study of the Liver (2017) EASL Clinical Practice Guidelines: the diagnosis and management of patients with primary biliary cholangitis. J Hepatol 67:145-172. https://doi.org/10.1016/j.jhep.2017.03.022

8. Hirschfield GM, Dyson JK, Alexander GJM Chapman MH, Collier J, Hübscher S, Patanwala I, Pereira SP, Thain C, Thorburn D, Tiniakos D, Walmsley M, Webster G, Jones DEJ (2018) The British Society of Gastroenterology/UKPBC primary biliary cholangitis treatment and management guidelines. Gut 67:1568-1594. https://doi.org/10.1136/gutjnl-2017-315259

9. Terziroli Beretta-Piccoli B, Mieli-Vergani G, Vergani D, Vierling JM, Adams D, Alpini G, Banales JM Beuers $U$, Björnsson $E$, Bowlus $C$, Carbone $M$, Chazouillères O, Dalekos G, De Gottardi A, Harada K, Hirschfield G, Invernizzi P, Jones D, Krawitt $E$, Lanzavecchia A, Lian Z-X, Ma X, Manns M, Mavilio D, Quigley EM, Sallusto F, Shimoda S, Strazzabosco M, Swain M, Tanaka A, Trauner M, Tsuneyama K, Zigmond E, Gershwin ME (2019) The challenges of primary biliary cholangitis: what is new and what needs to be done. J Autoimmun. https://doi.org/ 10.1016/j.jaut.2019.102328

10. Parés A, Caballería L, Rodés J (2006) Excellent long-term survival in patients with primary biliary cirrhosis and biochemical response to ursodeoxycholic Acid. Gastroenterology 130:715-720. https://doi.org/10.1053/j.gastro.2005.12.029

11. Murillo CFP, Harms MH, Lindor KD, van Buuren HR, Hirschfield GM, Corpechot C, van der Meer AJ, Feld JJ, Gulamhusein A, Lammers WJ, Ponsioen CY, Carbone $M$, Mason $A L$, Mayo $M J$, Invernizzi $P$, Battezzati PM, Floreani A, Lleo A, Nevens F, Kowdley KV, Bruns T, Dalekos GN, Gatselis NK, Thorburn D, Trivedi PJ, Verhelst $X$, Parés A Janssen HLA, Hansen BE, GLOBAL PBC Study Group (2020) Goals of treatment for improved survival in primary biliary cholangitis: treatment target should be Bilirubin within the normal range and normalization of alkaline Phosphatase. Am J Gastroenterol. https://doi.org/10.14309/ajg. 0000000000000557

12. Murillo CFP, Hirschfield GM, Corpechot C, Floreani $A$, Mayo $M J$, van der Meer $A$, Ponsioen $C Y$, Lammers WJ, Parés $A$, Invernizzi $P$, Carbone $M$, Battezzati PM, Nevens F, Kowdley KV, Thorburn D, Mason AL, Trivedi PJ, Lindor KD, Bruns T, Dale- 
kos GN, Gatselis NK, Verhelst X, Janssen HLA Hansen BE, Gulamhusein A, GLOBAL PBC Study Group (2019) Fibrosis stage is an independent predictor of outcome in primary biliary cholangitis despite biochemical treatment response. Aliment Pharmacol Ther 50:1127-1136. https://doi.org/10. 1111/apt.15533

13. Corpechot $C$, Chazouillères $O$, Rousseau $A$, Le Gruyer A, Habersetzer F, Mathurin P, Goria 0 , Potier P, Minello A, Silvain C, Abergel A, DebetteGratien M, Larrey D, Roux O, Bronowicki J-P, Boursier J, de Ledinghen $\mathrm{V}$, Heurgue-Berlot $\mathrm{A}$ Nguyen-Khac E, Zoulim F, Ollivier-Hourmand I, Zarski J-P, Nkontchou G, Lemoinne S, Humbert L, Rainteau D, Lefèvre G, de Chaisemartin L, CholletMartin S, Gaouar F, Admane F-H, Simon T, Poupon R (2018) A placebo-controlled trial of bezafibrate in primary Biliary Cholangitis. $\mathrm{N}$ Engl J Med 378:2171-2181. https://doi.org/10.1056/ NEJMoa1714519

14. de Vries E, Bolier R, Goet J, Parés A, Verbeek J, de Vree M, Drenth J, van Erpecum K, van Nieuwkerk K, van der Heide F, Mostafavi N, Helder J, Ponsioen C, Oude Elferink R, van Buuren $\mathrm{H}$, Beuers $\mathrm{U}$, Netherlands Association for the Study of the Liver-Cholestasis Working Group (2021) Fibrates for itch (FITCH) in Fibrosing Cholangiopathies: a double-blind, randomized, placebo-controlled trial. Gastroenterology 160:734-743.e6. https:// doi.org/10.1053/j.gastro.2020.10.001

15. Moctezuma-Velazquez C, Saffioti F, TasaycoHuaman S, Casu S, Mason A, Roccarina D, Vargas V, Nilsson J-E, Tsochatzis E, Augustin S, MontanoLoza AJ, Berzigotti A, Thorburn D, Genesca J, Abraldes JG (2019) Non-invasive prediction of high-risk varices in patients with primary Biliary Cholangitis and primary sclerosing cholangitis. Am J Gastroenterol 114:446-452. https://doi.org/10. 1038/s41395-018-0265-7

16. Karlsen $T H$, Folseraas $T$, Thorburn $D$, Vesterhus $M$ (2017) Primary sclerosing cholangitis - a comprehensive review. J Hepatol 67:1298-1323. https:// doi.org/10.1016/j.jhep.2017.07.022

17. Terziroli Beretta-Piccoli B, Vergani D, Mieli-Vergani G (2018) Autoimmune sclerosing cholangitis: evidence and open questions. J Autoimmun. https://doi.org/10.1016/j.jaut.2018.10.008

18. Dyson JK, Blain A, Shirley MDF, Hudson M, Rushton S, Jeffreys Jones DE (2021) Geo-epidemiology and environmental co-variate mapping of primary biliary cholangitis and primary sclerosing cholangitis. JHEP Rep 3:100202. https://doi.org/10.1016/ j.jhepr.2020.100202

19. European Society of Gastrointestinal Endoscopy, European Association for the Study of the Liver (2017) Role of endoscopy in primary sclerosing cholangitis: European Society of Gastrointestinal Endoscopy (ESGE) and European Association for the Study of the Liver (EASL) Clinical Guideline. J Hepatol 66:1265-1281. https://doi.org/10.1016/ j.jhep.2017.02.013

Publisher's Note. Springer Nature remains neutral with regard to jurisdictional claims in published maps and institutional affiliations.

\section{Aktuelle Buchempfehlungen in der Gastroenterologie}

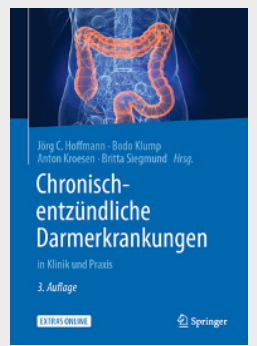

\section{Chronisch-entzündliche Darmerkrankungen}

Hoffmann, J.C., Klump, B., Kroesen, A., Siegmund, B. (Hrsg.) | 3. Aufl. 2020, XIV, 543 Seiten, 14 schwarz-weiß Abbildungen, 126 Abbildungen in Farbe, Hardcover 99,99€, ISBN 978-3-662-59103-1

Chronisch entzündliche Darmerkrankungen, mit den häufigsten Verlaufsformen Morbus Crohn und Colitis ulcerosa, haben an Häufigkeit in den letzten Jahrzehnten immer mehr zugenommen. Dieses Buch spannt einen Bogen von der Ursachenforschung über eine moderne Diagnostik mit Differentialdiagnostik bis hin zur Darstellung innovativer chirurgischer, internistischer und komplementärer Therapieformen. Die ausführliche Darstellung gibt Ärzt*innen verschiedener Fachrichtungen Gelegenheit, die jeweils anderen Therapien zu verstehen, um diese dann in das Therapiekonzept für jeden einzelnen Patient*innen sinnvoll zu integrieren. Dabei werden alle Aspekte sowohl der klinisch-stationären Versorgung als auch der langfristigen Betreuung im ambulanten Bereich abgedeckt.

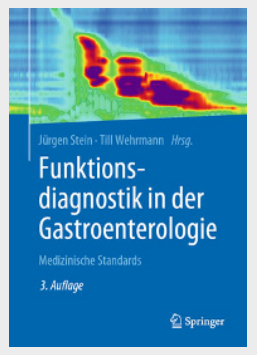

\section{Funktionsdiagnostik in der Gastroenterologie}

Stein, Jürgen, Wehrmann, Till (Hrsg.) | 3. Aufl. 2020, XI, 242 Seiten, 56 schwarz-weiß Abbildungen, 27 Abbildungen in Farbe, Softcover 59,99€, ISBN 978-3-662-59132-1

Funktionsdiagnostik auf einen Blick Für - Oberen und unteren Gastrointestinaltrakt - Pankreas - Leber und Gallenblase. Klar strukturiert und praxisrelevant - Prinzip der Untersuchungsmethode - Vorbereitung und technische Voraussetzungen - Durchführung und Ablauf - Auswertung und Befundinterpretation - Angaben zu Fehlerquellen, Störfaktoren, Referenzbereichen und Normwerten. Seit dem Erscheinen der letzten Auflage haben sich neue Untersuchungsmethoden etabliert und wissenschaftliche Erkenntnisse Eingang in den klinischen Alltag gefunden. Das bewährte Werk wurde daher vollständig aktualisiert, überarbeitet und entsprechend moderner Standards ergänzt.

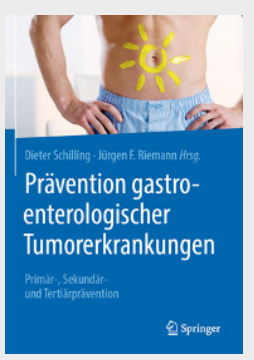

Prävention gastroenterologischer Tumorerkrankungen Schilling, Dieter, Riemann, Jürgen (Hrsg.)| 1 . Aufl. 2020, XV, 228 Seiten.,13 schwarz-weiß Abbildungen, 43 Abbildungen in Farbe, Softcover 59,99€, ISBN 978-3-662-58878-9

Tumoren des Magen-/Darmtrakts sind häufige Krebserkrankungen, die einerseits sehr hohe Kosten für das Gesundheitssystem bedingen, andererseits durch präventive Maßnahmen und Früherkennung in vielen Fällen nachgewiesenermaßen wirksam verhindert werden können. Eine Reihe von Screeningmethoden und diagnostischen Maßnahmen haben sich als effektiv erwiesen, hervorzuheben ist hier vor allem der Erfolg der Dickdarmkrebsvorsorge. Dieses Buch fasst zusammen, welche modernen und wirkungsvollen Möglichkeiten zur Prävention gastrointestinaler Tumoren derzeit eingesetzt werden, und zeigt auch auf, welche Herausforderungen, vor allem hinsichtlich einer effektiven Risikostratifikation und damit "Personalisierung”, noch zu meistern sind. Es wendet sich an Gastroenterolog*innen, Internist*innen ohne Schwerpunktbezeichnung, Allgemeinmediziner*innen/Hausärzt ${ }^{*}$ innen, Viszeralchirurg*innen sowie alle sonstigen medizinischen Zielgruppen, die sich für die Vorsorge gastrointestinaler Krebserkrankungen interessieren. 